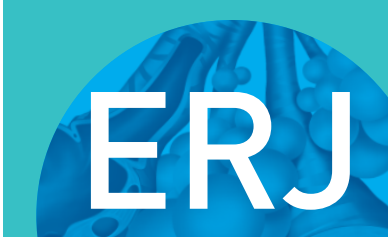

open research
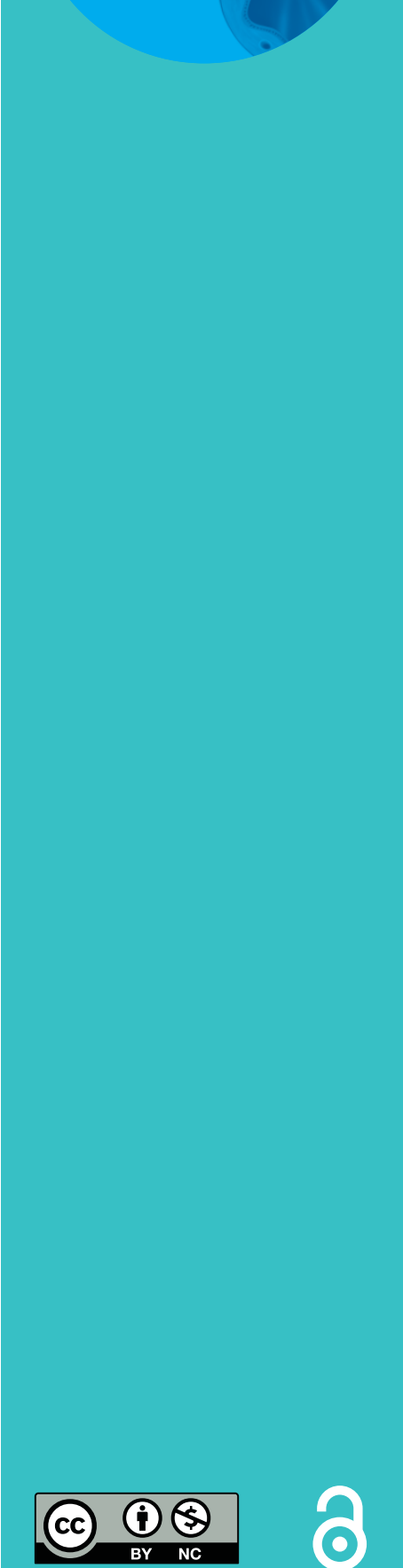

\section{Extended lifespan of bronchial epithelial cells maintains normal cellular phenotype and transcriptome integrity}

\author{
Jonathan O'Loughlin ${ }^{1,4}$, Robert J. Hall $\mathbb{1}^{1,4}$, Sangita Bhaker ${ }^{1}$, \\ Michael A. Portelli ${ }^{1}$, Amanda Henry ${ }^{1}$, Vincent Pang ${ }^{2}$, David O. Bates ${ }^{2}$, \\ Tyson V. Sharp ${ }^{3}$ and Ian Sayers ${ }^{1,4}$
}

Affiliations: ${ }^{1}$ Division of Respiratory Medicine, National Institute for Health Research, Nottingham Biomedical Research Centre, Biodiscovery Institute, University of Nottingham, Nottingham, UK. ${ }^{2}$ Division of Cancer and Stem Cells, School of Medicine, University of Nottingham, Biodiscovery Institute, Nottingham, UK. ${ }^{3}$ Centre of Cancer Cell and Molecular Biology, Barts Cancer Institute Queen Mary University of London, London, UK. ${ }^{4}$ These authors contributed equally.

Correspondence: Robert J. Hall, Division of Respiratory Medicine, National Institute for Health Research, Nottingham Biomedical Research Centre, Biodiscovery Institute, University of Nottingham, Nottingham, NG7 2RD, UK. E-mail: robert.hallanottingham.ac.uk

ABSTRACT Genetic studies have identified several epithelial-derived genes associated with airway diseases. However, techniques used to study gene function frequently exceed the proliferative potential of primary human bronchial epithelial cells (HBECs) isolated from patients. Increased expression of the polycomb group protein BMI-1 extends the lifespan of HBECs while maintaining cell context plasticity. Herein we aimed to assess how BMI-1 expression impacted cellular functions and global mRNA expression. HBECs from six donors were transduced with lentivirus containing BMI-1 and cells were characterised, including by RNA sequencing and impedance measurement. BMI-1-expressing HBECs (BHBECs) have a proliferative advantage and show comparable in vitro properties to low passage primary HBECs, including cell attachment/spreading and barrier formation. The B-HBEC mRNA signature was modestly different to HBECs, with only 293 genes differentially expressed (5\% false discovery rate). Genes linked to epithelial mesenchymal transition and cell cycle were enriched in B-HBECs. We investigated the expression of genes implicated in asthma from genetic and expression studies and found that $97.6 \%$ of genes remained unaltered. We have shown that increased BMI-1 expression in HBECs delays lung epithelial cell senescence by promoting cell cycle progression and highlighted the flexible utility for BHBECs as an important platform for studying airway epithelial mechanisms.

@ERSpublications

A method to extend the lifespan of primary human bronchial epithelial cells that maintain a normal epithelial cell phenotype, thus providing a platform to investigate respiratory disease mechanisms over prolonged periods https://bit.ly/353Rklc

Cite this article as: O'Loughlin J, Hall RJ, Bhaker S, et al. Extended lifespan of bronchial epithelial cells maintains normal cellular phenotype and transcriptome integrity. ERJ Open Res 2020; 7: 002542020 [https://doi.org/10.1183/23120541.00254-2020].

\section{The reagents outlined to generate BMI-1 cells are freely available to all researchers on request.}

This article has supplementary material available from openres.ersjournals.com.

Received: 7 May 2020 | Accepted after revision: 17 Aug 2020

Copyright $\odot$ ERS 2020. This article is open access and distributed under the terms of the Creative Commons Attribution Non-Commercial Licence 4.0. 


\section{Introduction}

The proximal airway epithelium is a critical defensive barrier that protects underlying tissues from inhaled pathogens and environmental particles by orchestrating innate and adaptive immune responses. In chronic airway diseases such as asthma there is growing evidence that the airway epithelium is fundamentally different in a number of aspects, including the epigenome and transcriptome [1], cell composition [2], and barrier properties [3]. Genome-wide association studies (GWASs) and RNA-sequencing studies have identified numerous genetic variants (e.g. within IL33, TSLP gene loci $[4,5])$ and differentially expressed genes of relevance to the airway epithelium (CEACAM5, CPA3, INSR, MS4A2, NOX1, and SLC16A6 [6]). Many genes identified from these studies are novel and thus their contribution to airway disease have yet to be elicited and importance defined.

Culturing primary human bronchial epithelial cells (HBECs) isolated from healthy controls or asthmatic patients at the air-liquid interface (ALI) is the gold standard for studying physiologically relevant lung epithelial biology [7]. However, the short lifespan (3-4 passages) of primary airway epithelial cells when cultured in vitro, is insufficient for many in vitro techniques such as CRISPR/cas9 gene editing and single cell cloning prior to ALI. Thus, there is a need for a cell culture model with an enhanced lifespan potential, whilst retaining the plasticity and phenotype of primary epithelial cells. This is particularly important as the bronchoscopy procedure to isolate cells has significant risks to the donor.

There have been numerous different approaches to resolve primary epithelial cell senescence, including culturing cells with Rho-associated protein kinase (ROCK) inhibitors, seeded on a layer of irradiated feeder cells [8]. Whilst such methods allow normal epithelial cells to proliferative indefinitely in vitro, it is complex and time-consuming when compared to standard cell culture methods. Alternative methods have overexpressed proteins such as human telomerase reverse transcriptase (hTERT) within cells to successfully extend their proliferative potential [9]. However, hTERT-expressing cells have been shown to lose the ability to differentiate at early passage and be genetically unstable $[9,10]$; two key elements required comparable to wild type cells for in vitro disease research.

B-cell-specific Moloney murine leukaemia virus integration site 1 (BMI-1) is a polycomb group protein that is critical in regulating cell senescence through suppression of the cyclin-dependent kinase inhibitor, p16 ${ }^{\mathrm{INK} 4 \mathrm{~A}}[11,12]$. We have shown that cells engineered to overexpress BMI-1 retain key phenotypes of primary bronchial epithelial cell cultures up to passage 15 , including the ability to differentiate at the ALI and a normal karyotype, which has been robustly replicated by others [10, 13]. However, all of these studies have lacked the fundamental understanding of the global gene expression profile that is manipulated, deregulated and controlled in the context of BMI-1 overexpression. This is essential if we are to expand and effectively utilise this important lung cell culture platform for basic and disease biology.

In this study, we have addressed the previously uncharacterised biology of this lung cell model system. We have 1) evaluated the BMI1-HBEC (B-HBEC) system in detail as a platform representative of primary human airway epithelium at both the cellular and molecular level; 2) developed an inducible BMI-1 system that can be silenced allowing a research platform directly comparable to low passage donor cells; and 3 ) evaluated the utility of the platform to investigate genes implicated in asthma.

\section{Materials and methods}

\section{Primary bronchial epithelial cell culture}

Normal HBECs (N-HBECs) were purchased from Lonza (Wokingham, UK) (supplementary table S1). $\mathrm{N}-\mathrm{HBEC}$ were grown in BEGM (Lonza) supplemented with $13 \mathrm{mg} \cdot \mathrm{mL}^{-1}$ bovine pituitary extract, $0.5 \mathrm{mg} \cdot \mathrm{mL}^{-1}$ hydrocortisone, $0.5 \mu \mathrm{g} \cdot \mathrm{mL}^{-1}$ human recombinant epidermal growth factor, $0.5 \mathrm{mg} \cdot \mathrm{mL}^{-1}$ epinephrine, $10 \mathrm{mg} \cdot \mathrm{mL}^{-1}$ transferrin, $5 \mathrm{mg} \cdot \mathrm{mL}^{-1}$ insulin, $0.1 \mu \mathrm{g} \cdot \mathrm{mL}^{-1}$ retinoic acid, $6.5 \mu \mathrm{g} \cdot \mathrm{mL}^{-1}$ triiodothyronine, $30 \mu \mathrm{g} \cdot \mathrm{mL}^{-1}$ gentamicin and $5 \mathrm{ng} \cdot \mathrm{mL}^{-1}$ amphotericin as previously described [7].

\section{Production of genetically modified HBECs}

Full length human BMI-1 was amplified and cloned into a pLVX-Puro constitutive vector (Clontech) and a pCDH-CuO-MCS-IRES-RFP (SparQ) inducible vector (System Biosciences). pLVX-Puro-BMI-1, SparQ-BMI-1 and pCDH-EF1-CymR-T2A-Puro (System Biosciences) vectors were packaged into pCMV delta R8.9 and pCMV-VSV-G lentivirus envelope and packaging vectors. Passage $2 \mathrm{~N}$-HBECs from six donors were transduced with pLVX-Puro-BMI-1 lentivirus solution to produce HBECs that constitutively express BMI-1 (B-HBEC), as described previously [10]. These B-HBECs were continued in culture through additional passages 3, 4, 5 and 6. To generate an inducible BMI-1 cell line, N-HBECs from five donors were transduced with SparQ-BMI-1 lentivirus solution and pCDH-EF1-CymR-T2A-Puro lentivirus solution (1:3 ratio). Additional details are described in the supplementary material. 
MTT assay

A total of $2.5 \times 10^{3}$ cells were seeded onto a 96-well plate in $200 \mu \mathrm{L}$ of BEGM. Cells were incubated for 72 or $96 \mathrm{~h}$. Medium was replaced with $200 \mu \mathrm{L} 0.5 \mathrm{mg} \cdot \mathrm{mL}^{-1}$ 3-(4,5-dimethylthiazol-2-yl)-2,5-diphenyltetrazolium bromide (MTT) dissolved in BEGM. Cells were incubated with MTT at $37^{\circ} \mathrm{C}$ for $4 \mathrm{~h}$. MTT solution was replaced with $200 \mu \mathrm{L}$ isopropanol and incubated for $10 \mathrm{~min}$. The absorbance of the solution was measured at $570 \mathrm{~nm}$ (subtracting the $690 \mathrm{~nm}$ background) using a Flexstation 3 microplate reader (Molecular Devices, Wokingham, UK).

\section{DAPI staining}

Cells suspended in BEGM were transferred into a Via-1 Cassette, lined with acridine orange and 4',6-diamidino-2-phenylindole (DAPI) and analysed on a Nucleocounter NC-3000 (Chemometec, Allerod, Denmark).

\section{Electric cell-substrate impedance sensing}

The $8 \mathrm{~W} 10 \mathrm{E}$ arrays (IBIDI, Munich, Germany) were treated with $200 \mu \mathrm{L} 10 \mathrm{mM}$ L-cysteine dissolved in sterile water for $15 \mathrm{~min}$ at room temperature. Wells were washed $3 \times$ with $300 \mu \mathrm{L}$ sterile water. Then, $200 \mu \mathrm{L}$ PureCol Type 1 bovine collagen solution (Advanced Biomatrix, San Diego, CA, USA) diluted 1 in 100 in sterile water, was added to wells and incubated at $37^{\circ} \mathrm{C}$ overnight. Wells were washed $3 \times$ with BEGM. Then, $400 \mu \mathrm{L}$ BEGM was added to each well and the array was stabilised in the electric cell-substrate impedance sensing (ECIS) Z $\theta$ instrument (Applied Biophysics, Troy, NY, USA). Medium was replaced with $7.5 \times 10^{4}$ cells in BEGM. The array was run at multiple frequencies (including the specific frequencies of $400 \mathrm{~Hz}$ and $32 \mathrm{kHz}$ ) for $72 \mathrm{~h}$ continuously on the ECIS $\mathrm{Z} \theta$ instrument, as described previously [14].

Two-step cell cycle assay

A total of $4 \times 10^{5}$ cells were centrifuged at $400 \times \mathrm{g}$ for $5 \mathrm{~min}$. Pellets were washed with PBS then resuspended in $100 \mu \mathrm{L}$ lysis buffer (Chemometec) containing $10 \mu \mathrm{g} \cdot \mathrm{mL}^{-1}$ DAPI (Chemometec) and incubated at $37^{\circ} \mathrm{C}$ for $5 \mathrm{~min}$. Then, $100 \mu \mathrm{L}$ stabilisation buffer (Chemometec) was added to the solution and $30 \mu \mathrm{L}$ of sample was transferred into an NC-Slide A2 (Chemometec). A two-step cell cycle assay was run on a Nucleocounter NC-3000 (Chemometec). Data were exported as an FCS file and analysed using FlowJo software. The cell cycle distribution for 10000 cells was automatically quantified using the cell cycle modelling algorithm as described [15].

\section{RNA extraction and isolation}

Cells were grown in T75 flasks until $80 \%$ confluent. RNA was isolated using an RNeasy Mini Kit (QIAGEN, Manchester, UK) following the manufacturer's protocol. RNA integrity number was assessed using the 2100 Bioanalyser (Agilent, Didcot, UK).

\section{RNA sequencing}

Library preparation is described in the supplementary material. Paired-end (75 bp) sequencing was performed using a HiSeq4000 platform, HiSeq 3000/4000 PE Cluster kit and 150 cycle SBS kit (Illumina, Cambridge, UK), generating a raw read count of $\sim 30$ million reads per sample.

\section{Analysis of RNA sequencing}

Alignment of RNA-sequencing reads to genome build GRCh37 was carried out using TopHat package [16]. Analysis of RNA-sequencing files was performed using the Cufflinks package [16] as performed previously [17]. Principal-component analysis (PCA) was completed using the R package prcomp using FPKM values generated by Cuffnorm and PCA plots were produced using ggplot2. Additional details are described in the supplementary material.

\section{Lookup of asthma-related genes}

A lookup of asthma-related genes was performed in the generated RNA-sequencing datasets. Asthma-related genes were identified from the most recent and largest asthma GWAS [5] and from meta-analyses of gene expression in bronchial epithelial brush samples from asthma patients and controls [6].

\section{Statistical analysis}

For cell-based outcomes, a paired t-test was used to determine differences between N-HBECs and B-HBECs. To determine whether BMI-1 expression altered cell cycle phase frequency, a two-way ANOVA with Sidak's multiple comparisons test was performed. $\mathrm{p}<0.05$ was considered significant. 


\section{Results}

BMI-1 does not change the viability of HBECs

To determine the effects of BMI-1 upregulation on cell viability, an MTT assay was performed. The cell viability of passage 3 (P3) N-HBECs were compared to passage 6 (P6) B-HBECs from six donors (supplementary table S1). At both 72 and $96 \mathrm{~h}$, there was a nonsignificant increase in cell viability in five of six B-HBEC donors (figure 1a and b). Staining with DAPI to assess cell viability also showed no difference between B-HBECs and N-HBECs populations (figure 1c).

\section{BMI-1 expression alters cell cycle distribution of HBECs}

We observed a significant decrease of $12 \%$ in the frequency of B-HBECs within the G1/G0 phase $(p<0.001)$ and an increase of $9 \%$ in $G 2 / M$ phase cells $(p<0.01)$ when compared to N-HBECs (figure 1d and supplementary figure $\mathrm{S} 1$ ).

\section{BMI-1 does not impact cell spreading or tight junction formation in HBECs}

To assess how BMI-1 overexpression impacted cell adherence, spreading or tight junction formation we performed ECIS. In all comparisons of N-HBEC versus B-HBEC no difference was observed for capacitance (cell spreading/adherence) or resistance (tight junction formation), highlighting that B-HBECs are not different from N-HBECs in these properties (figure 2).

\section{Constitutive overexpression of BMI1 in N-HBECs produces a transcriptional response}

To determine how BMI-1 expression affected HBECs at the transcriptome level, RNA sequencing was performed using RNA isolated from early passage (P2) N-HBECs and P5 B-HBECs (figure 3a). PCA of $\mathrm{N}$-HBECs and B-HBECs identified significant overlap in the sample datasets with no obvious outliers (supplementary figure S2). We first confirmed that all six HBECs donors transduced with pLVX-BMI-1 had significant upregulation of BMI-1 transcripts in comparison to N-HBECs (figure $3 \mathrm{~b} ; \mathrm{p}<0.0001$ ). Of
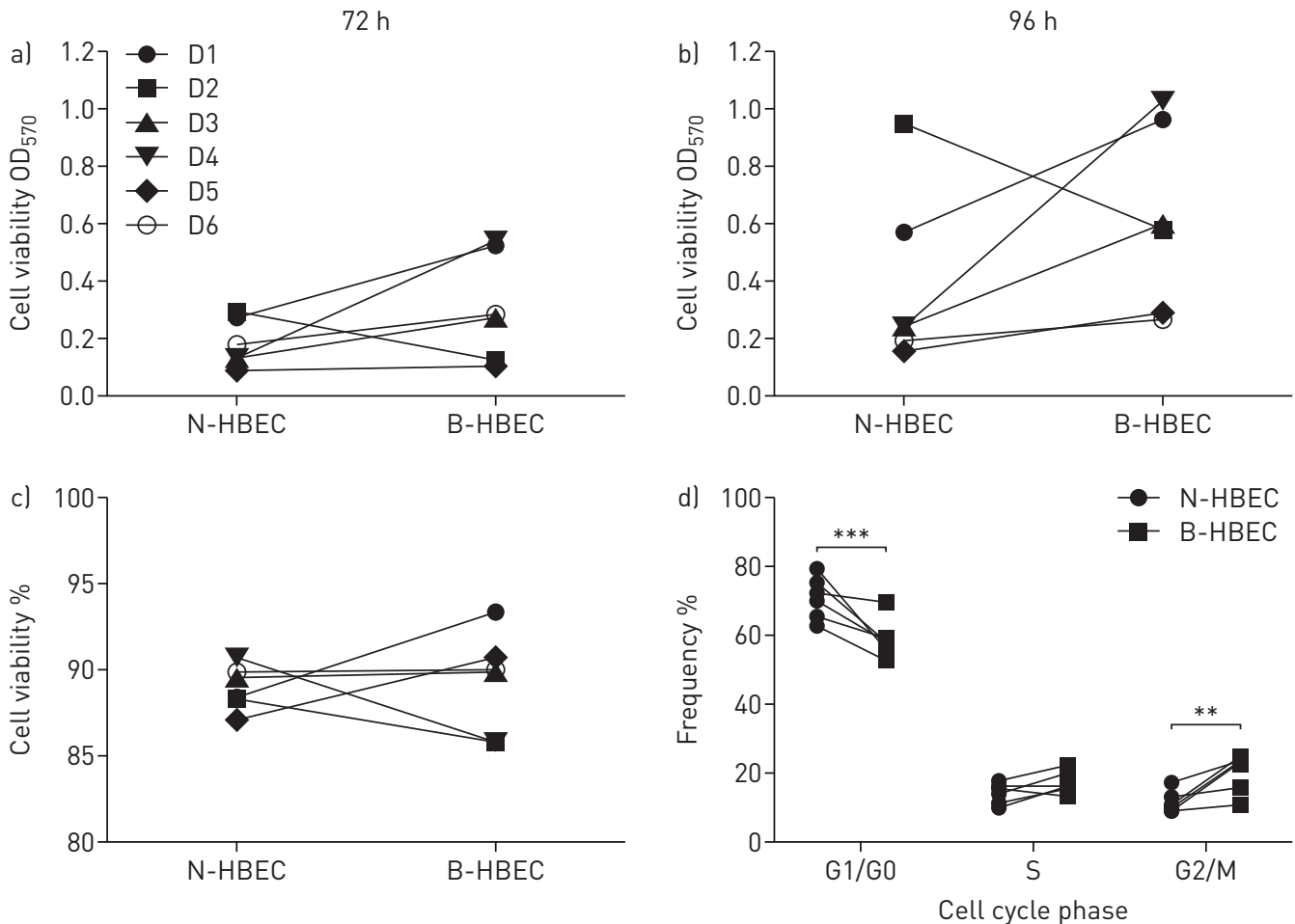

FIGURE 1 Phenotypic properties of BMI-1-expressing human bronchial epithelial cells (B-HBECs) and normal human bronchial epithelial cells (N-HBECs). Basic cell phenotypes of passage $3 \mathrm{~N}$-HBECs and passage 6 B-HBECs derived from the same matched donors. $a$ and b) An MTT (3-(4,5-dimethylthiazol-2-yl)-2,5diphenyltetrazolium bromide) assay was performed to assess cell viability; cells were incubated for $72 \mathrm{~h} \mathrm{(a)}$ and $96 \mathrm{~h}(\mathrm{~b})$ before the addition of MTT. c) Cell viability determined through DAPI staining and counted on a Chemometec Nucleocounter NC-3000. d) The cell cycle phase distribution in N-HBECs and B-HBECs determined through a two-step cell cycle assay with cell cycle phase frequency, calculated using the Watson pragmatic algorithm. Values represent the median and $95 \%$ confidence interval $(n=6)$. A paired $t$-test $(a-c)$, two-way ANOVA and Sidak's multiple comparison test (d) were used to determine statistical significance. ${ }^{* *}$ : $p<0.01 ;{ }^{* * *}: p<0.001$ 

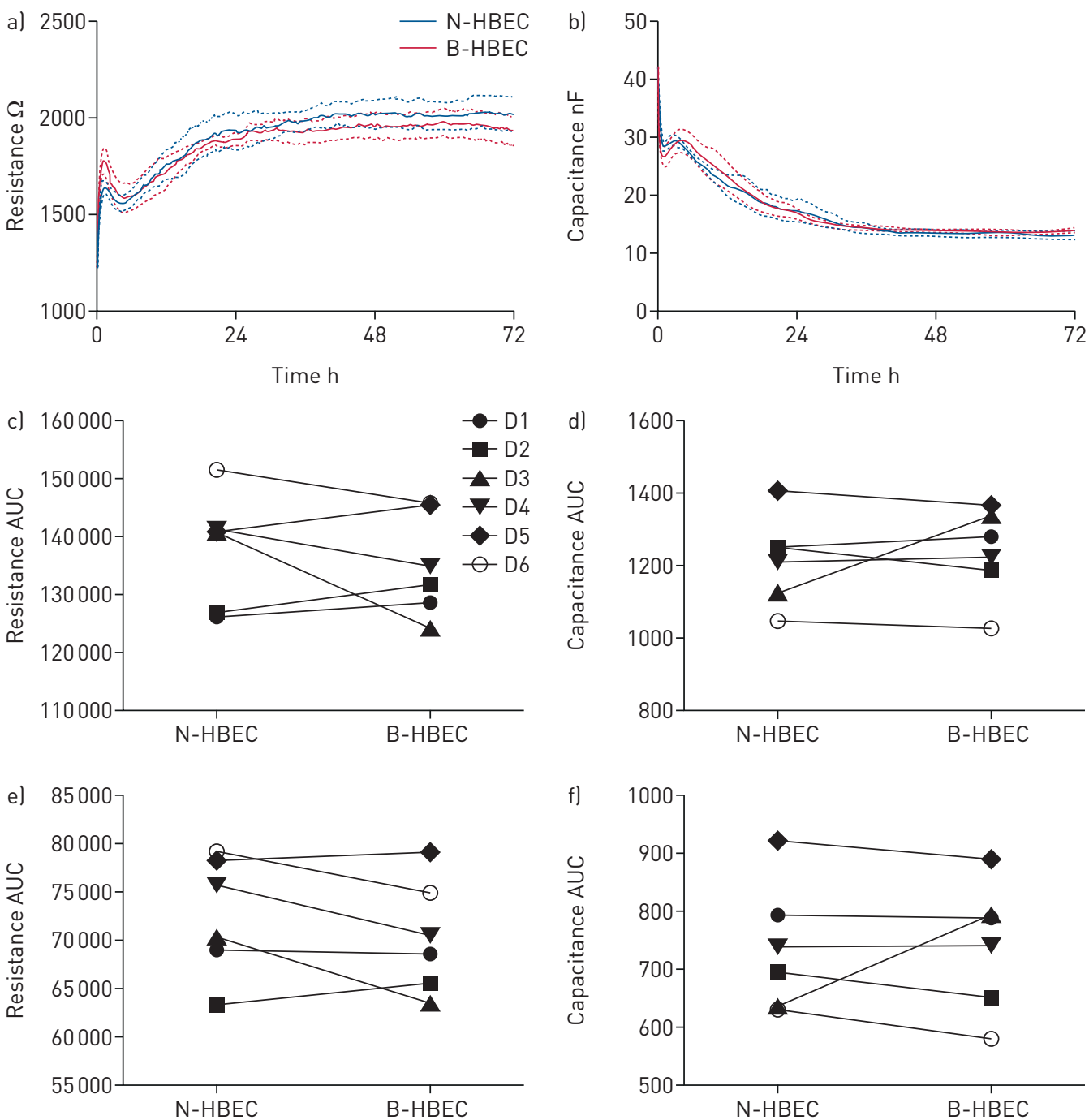

FIGURE 2 The effects of BMI-1 on human bronchial epithelial cell (HBEC) adherence, spreading and tight junction formation, in vitro. Passage 3 normal HBECs (N-HBECs) and passage 6 BMl-1-expressing HBECs (B-HBECs) were seeded on an 8W10E array (IBIDI) and cultured for $72 \mathrm{~h}$. Resistance was measured at $400 \mathrm{~Hz}$ (barrier formation) and capacitance at $32 \mathrm{kHz}$ (cell adherence and spreading). Raw resistance (a) and capacitance (b) profile of N-HBECs (blue) and B-HBECs (red), values represent mean and SEM ( $n=6)$. Total area under the curve from resistance (c) and capacitance (d) profile over $72 \mathrm{~h}$. Area under the curve from resistance profile at $36-72 \mathrm{~h} \mathrm{(e)} \mathrm{and} \mathrm{capacitance} \mathrm{at} 0-36 \mathrm{~h}(\mathrm{f})$. A paired $t$-test was used to determine statistical significance $(c-f)$.

the 19190 transcripts evaluated, 293 genes (1.5\%) were differentially expressed at 5\% false discovery rate (FDR). There were 161 genes that were upregulated and 132 were downregulated in B-HBECs compared to $\mathrm{N}-\mathrm{HBECs}$ (figure $3 \mathrm{c}$ ). The top 10 upregulated genes based on magnitude of effect and p-value were; GUSBP2, HAS2, THY1, DENND2A, TOX2, CSF3, CPNE7, ATP1A3, BCL2A1, KIAA1644 and those downregulated were; COX7A1, HEATR6, SPINK7, ATP12A, PROSP, MUC15, SPRR3, CALB1, SBSN, KRT13 (table 1 and figure $3 \mathrm{c}$ ). We observed multiple changes to extracellular matrix proteins, including increases in collagen genes (COL4A1, COL4A2, COL22A1) and decreases in cytokeratin genes (KRT4, KRT10, KRT13, KRT16, KRT23) in B-HBECs (supplementary table S2 for complete list). A heat map was generated using genes with a five-fold or greater change in expression, demonstrating that there was heterogeneity at the donor level. However, the expression profiles of N-HBECs and B-HBECs are visibly distinct for these highest differentially expressed genes (figure $3 \mathrm{~d}$ ).

\section{Gene set enrichment analysis shows that genes involved in cell cycle regulation are altered in} $B-H B E C S$

To provide an insight into how BMI-1 expression affected the biological processes of bronchial epithelial cells we used gene set enrichment analysis. A total of 143 pathways were enriched in B-HBECs 

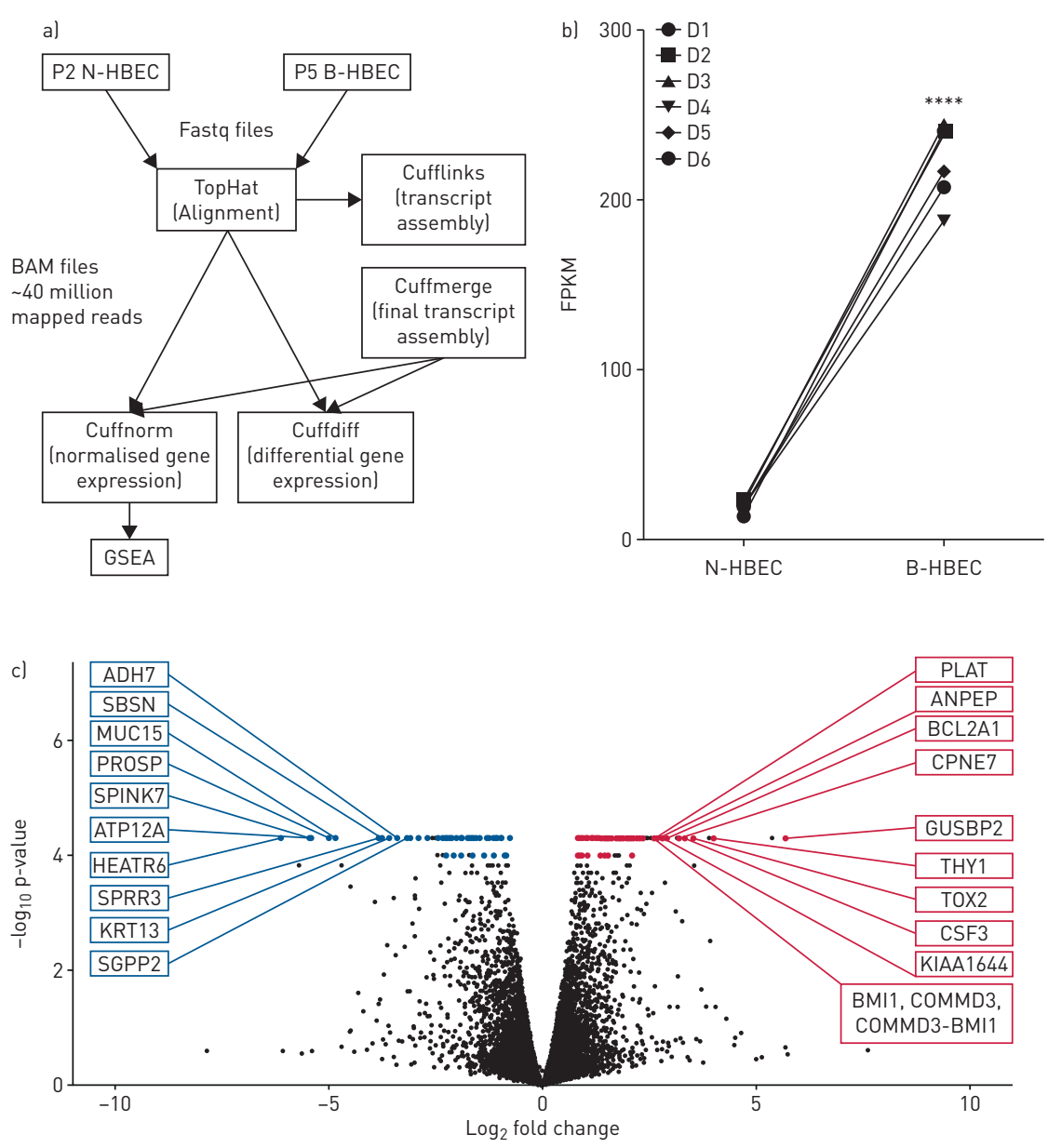

d)

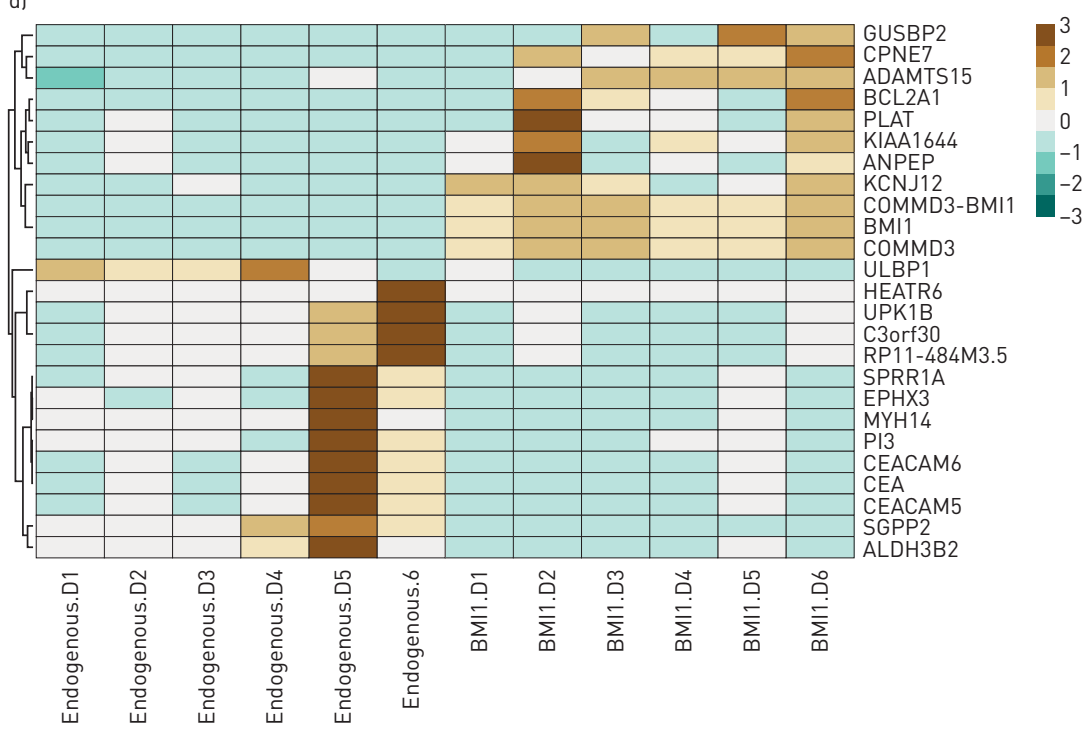

FIGURE 3 RNA sequencing analysis of BMI-1-expressing human bronchial epithelial cells (B-HBECs) versus normal human bronchial epithelial cells (N-HBECs). a) Schematic showing the RNA-sequencing pipeline. Scythe/Sickle trimmed Fastq files (.fastq) were aligned to the GRCh37 human genome assembly to produce BAM files (.bam) with an average of $\sim 40$ million mapped reads using the TopHat software package. Cufflinks and Cuffmerge were used for transcript assembly and Cuffnorm and Cuffdiff were used for normalised and differential gene expression, respectively. b) Total BMI-1 expression (FPKM) in P2 N-HBECs and P5 B-HBECs. Statistical analysis was carried out using a paired t-test (****: $p<0.0001)$. c) 293 genes were differentially expressed between B-HBECs and N-HBECs with 132 downregulated and 161 upregulated $(5 \%$ false discovery rate). Coloured dots represent differentially expressed genes $(\mathrm{q}<0.05)$ and labelled genes are the top 10 differentially expressed genes ranked firstly on q-value then on fold change. d) Genes that showed a $>5$-fold change in expression ( $q=0.006)$. Red represents over expressed, and blue attenuated genes. 
(upregulated by BMI-1) whilst 20 pathways were enriched in N-HBECs (downregulated by BMI-1) at 5\% FDR (supplementary table S3). The most upregulated pathways in B-HBECs consisted of genes involved in epidermal growth factor receptor (EGFR) signalling (normalised enrichment score (NES) 2.23), cell-cycle regulated genes (NES 2.06 and 2.04) and epithelial mesenchymal transition (EMT) (NES 2.02) (table 2). The top downregulated pathway in B-HBECs involved genes in intestinal cells in response to co-culture with probiotic bacteria (NES -2.11) and mucociliary differentiation (NES -2.01). These gene and pathway data 1) indicate that BMI-1 expression induces changes in the expression profile of several pathways and 2) provide an expression map for researchers interested in using this platform for specific pathway-related questions.

\section{BMI-1 expression in an inducible model is not reversible and shows heterogeneity}

In addition to the constitutive B-HBEC cell line, we developed a second-generation cell system with inducible BMI-1-expression HBECs (IB-HBECs) controlled through a cumate switch. We confirmed that IB-HBECs cultured in the presence of cumate have significantly upregulated BMI-1 transcripts $\left({ }^{*} \mathrm{p}<0.05\right)$. However, withdrawal of cumate had either no effect or a modest decrease on BMI-1 expression levels (figure 4a). Overall, 104 genes out of 16384 assayed (0.6\%), were differentially expressed between $\mathrm{N}-\mathrm{HBEC}$ and IB-HBECs cultured in the presence of cumate (supplementary table S4). This is markedly fewer than we observed in the constitutive B-HBEC system (figure $4 \mathrm{~b}$ ). Of these, 74 genes were upregulated, whilst 30 genes were downregulated in comparison to N-HBECs. The top 10 upregulated genes included: NES, S100A7, TSPAN18, NTRK1, BMI1/COMMD3, ANPEP, B4GALNT1, COL8A2, CA14 and HEPHL1 and downregulated genes included: CLCA4, AC005702.1/AC005702.4, KRT13, KRT4, MSMB, NDN, ATP12A, CRNN, ALDH3A1 and METTL7A ranked as previously (figure 4b). It is evident that

TABLE 1 Top 10 upregulated and downregulated genes in B-HBECs (P5) compared to N-HBECs (P2) ranked firstly on q-value then by fold change $(q<0.01$ for all $)$

\begin{tabular}{|c|c|c|}
\hline Gene & $\begin{array}{l}\text { Direction/fold } \\
\text { change }\end{array}$ & Description \\
\hline GUSBP2 & +52 & Pseudogene. \\
\hline HAS2 & +42 & $\begin{array}{l}\text { Hyaluronan synthase } 2 \text {. Hyaluronan or hyaluronic acid is a constituent of the extracellular matrix. It serves a } \\
\text { variety of functions, including space filling, lubrication of joints and provision of a matrix through which } \\
\text { cells can migrate. }\end{array}$ \\
\hline THY1 & +16 & $\begin{array}{l}\text { Thy- } 1 \text { cell surface antigen. The encoded protein is involved in cell adhesion and cell communication in } \\
\text { numerous cell types, but particularly in cells of the immune and nervous systems. }\end{array}$ \\
\hline DENND2A & +15 & $\begin{array}{l}\text { DENN domain containing } 2 A \text {. Guanine nucleotide exchange factor, which may activate RAB9A and RAB9B. } \\
\text { Promotes the exchange of GDP to GTP, converting inactive GDP-bound Rab proteins into their active } \\
\text { GTP-bound form. }\end{array}$ \\
\hline TOX2 & +12 & $\begin{array}{l}\text { TOX high mobility group box family member } 2 \text {. Gene Ontology annotations related to this gene include } \\
\text { chromatin binding and RNA polymerase II transcription factor binding. }\end{array}$ \\
\hline CSF3 & +10 & $\begin{array}{l}\text { Colony stimulating factor } 3 \text {. The protein encoded by this gene is a cytokine that controls the production, } \\
\text { differentiation and function of granulocytes. }\end{array}$ \\
\hline CPNE7 & +9 & Copine 7. Calcium-dependent membrane-binding proteins. \\
\hline ATP1A3 & +9 & $\begin{array}{l}\text { ATPase } \mathrm{Na}+/ \mathrm{K}+\text { transporting subunit } \alpha 3 \text {. The protein encoded by this gene belongs to the family of } \mathrm{p} \text {-type } \\
\text { cation transport ATPases, and to the subfamily of } \mathrm{Na}+/ \mathrm{K}+- \text {-ATPases. }\end{array}$ \\
\hline$B C L 2 A 1$ & +8 & $\begin{array}{l}\text { BCL2 Related protein A1. This gene encodes a member of the BCL-2 protein family. The proteins of this } \\
\text { family form hetero- or homodimers and act as anti- and proapoptotic regulators that are involved in a wide } \\
\text { variety of cellular activities such as embryonic development, homeostasis and tumourigenesis. }\end{array}$ \\
\hline KIAA1644 & +7 & Unknown function. \\
\hline COX7A1 & -Infinite & $\begin{array}{l}\text { Cytochrome } \mathrm{C} \text { oxidase subunit } 7 \mathrm{~A} 1 \text {. Cytochrome } \mathrm{c} \text { oxidase, the terminal component of the mitochondrial } \\
\text { respiratory chain, catalyses the electron transfer from reduced cytochrome } \mathrm{c} \text { to oxygen. }\end{array}$ \\
\hline HEATR6 & -70 & Diseases associated with HEATR6 include breast cancer. \\
\hline SPINK7 & -44 & Serine peptidase inhibitor, Kazal type 7. Probable serine protease inhibitor. \\
\hline ATP12A & -43 & $\begin{array}{l}\text { ATPase } \mathrm{H}+/ \mathrm{K}+\text { transporting nongastric } \alpha 2 \text { subunit. The protein encoded by this gene belongs to the family of } \\
\text { P-type cation transport ATPases. }\end{array}$ \\
\hline PROSP & -32 & Pseudogene. \\
\hline MUC15 & -29 & Mucin 15. Cell surface Associated. May play a role in the cell adhesion to the extracellular matrix. \\
\hline SPRR3 & -14 & Small proline rich protein 3 . Among its related pathways are keratinisation and developmental biology. \\
\hline CALB1 & -14 & Calbindin 1. This protein is thought to buffer entry of calcium upon stimulation of glutamate receptors. \\
\hline SBSN & -13 & Suprabasin. Diseases associated with SBSN include adenoid cystic carcinoma. \\
\hline KRT13 & -12 & $\begin{array}{l}\text { Keratin 13. The keratins are intermediate filament proteins responsible for the structural integrity of } \\
\text { epithelial cells and are subdivided into cytokeratins and hair keratins. }\end{array}$ \\
\hline
\end{tabular}

N-HBEC: normal human bronchial epithelial cell; B-HBEC: BMI-1-expressing human bronchial epithelial cells. 
TABLE 2 Gene set enrichment analysis was carried out to identify differentially regulated pathways between P2 N-HBECs and P5 B-HBECs

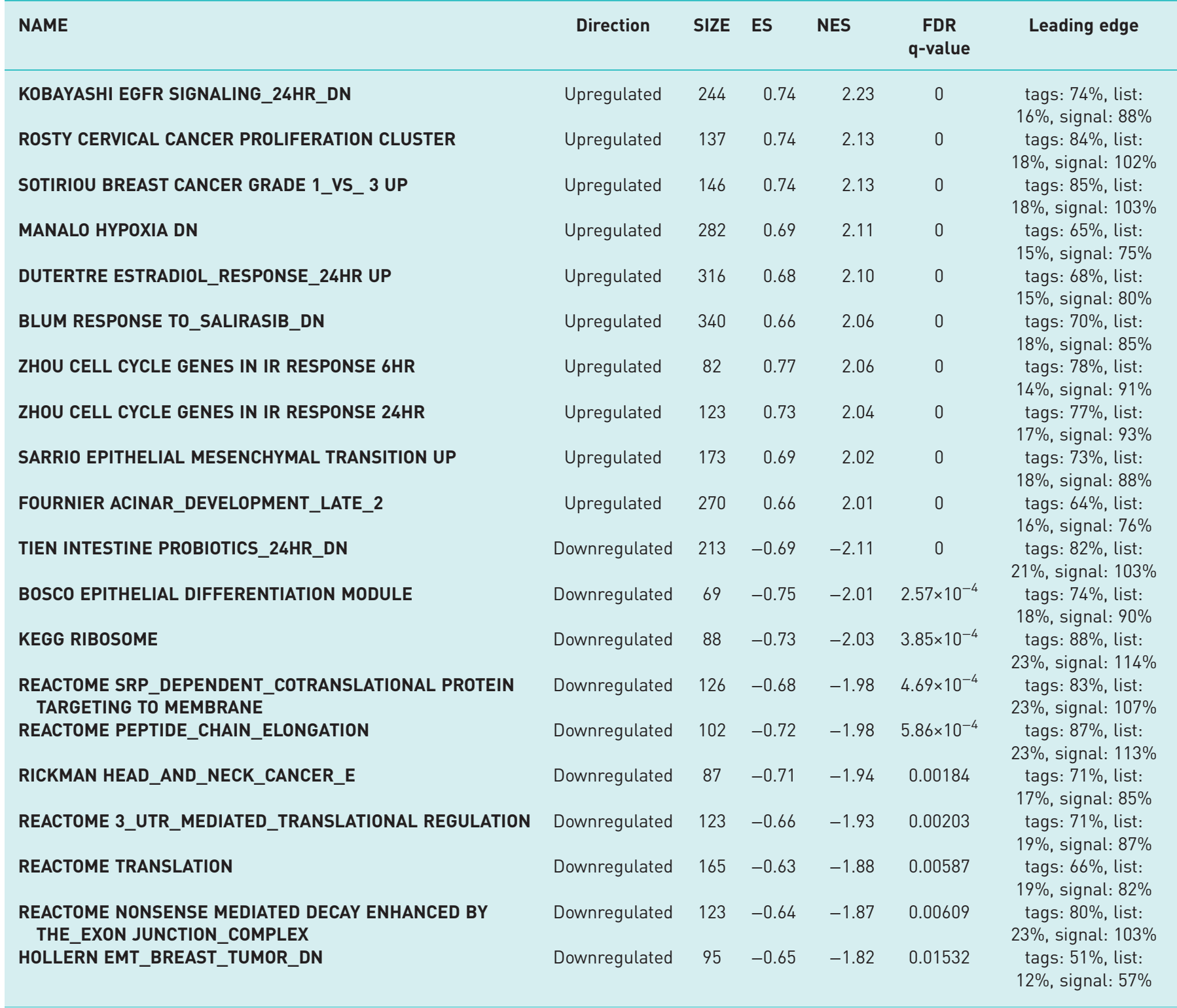

143 pathways were significantly $(q<0.05)$ enriched in B-HBECs and 20 were enriched in the N-HBECs (top 10 shown ranked on q-value and then ES). Size indicates the number of genes in the gene set after filtering out those not in the expression dataset.N-HBEC: normal human bronchial epithelial cell; B-HBEC: BMI-1-expressing human bronchial epithelial cells; ES: enrichment score for the gene set, which is the degree that the gene set is over-represented at the top or bottom of the ranked list of genes in the expression dataset; NES: normalised enrichment score; FDR q-value: false discovery rate adjusted p-value, which is the estimated probability that the NES represents a false positive; leading edge subset: subset of members that contribute the most to the ES; tags: percentage of genes contributing to the ES.

N-HBECs have a distinct expression profile compared to IB-HBECs and that there is no distinct difference between IB-HBECs that have BMI-1 expression switched on (+cumate) and IB-HBECs that have BMI-1 silenced (-cumate) (figure 4c and supplementary table S5). Finally, we compared the expression profile of constitutive and inducible B-HBECs, of the total 293 genes that were differentially expressed between B-HBECs and N-HBECs, just 20 of those genes were also differentially expressed between IB-HBECs and N-HBECs (figure 4d).

BMI-1 constitutive expressing cell platform has a physiologically valid utility for asthma research To begin to address the utility of the BMI-1 constitutive cell lines for asthma research we investigated the expression profile of genes implicated in asthma in the most recent and largest GWAS [5] and in a 

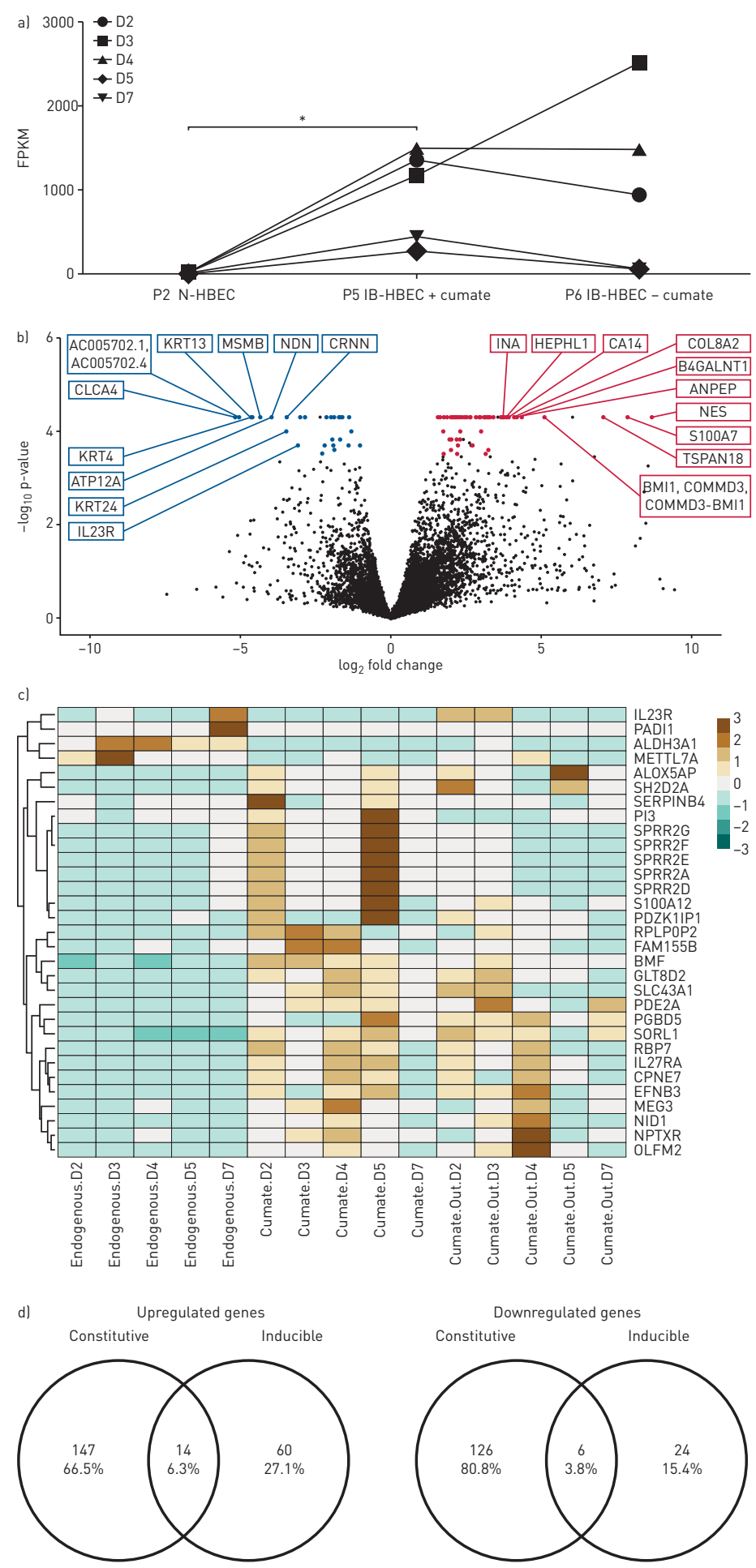

FIGURE 4 RNA sequencing analysis of inducible BMI-1-expressing human bronchial epithelial cells (B-HBECs) cultured with and without cumate. a) Total BMI-1 expression (FPKM) in P2 normal human bronchial epithelial cells (N-HBECs), P5 inducible HBECs (IB-HBECs) in the presence of cumate and P6 IB-HBECs with cumate washed out. Statistical analysis carried out with a Friedman test with Dunn's multiple comparisons test $(*: p<0.05)$. b) 104 genes are differentially expressed between BMI-1-expressing and endogenous cells with 74 genes upregulated and 30 genes downregulated ( $5 \%$ false discovery rate). Coloured dots reached $\mathrm{q}<0.05$ and labelled genes are the top 10 differentially expressed genes ranked firstly on q-value then fold change. c) Genes that showed a $>5$-fold change in expression ( $q=0.006$ ) between IB-HBECs and $\mathrm{N}-\mathrm{HBEC}$, including the expression levels after cumate withdrawal. Red represents high expression and blue low. d) Venn diagram showing the proportion of genes that are differentially expressed in both the constitutive and inducible B-HBEC systems. 
meta-analyses of gene expression in bronchial epithelial brush samples from asthma patients and controls [6]. We identified that out of the 289 genes implicated in these studies that had quantitative data available, 282 (97.6\%) were not altered in B-HBECs versus N-HBECs (supplementary table S6).

\section{Discussion}

In this study we aimed to 1) evaluate the B-HBEC system in detail as a platform representative of primary human airway epithelium at both the cellular and transcriptional level; 2) develop an IB-HBEC system that can be silenced allowing a research platform with extended lifespan and directly comparable to low passage donor cells; and 3) evaluate the utility of the platform to investigate genes implicated in asthma.

Our data demonstrate that B-HBECs retain the cellular phenotype of primary N-HBECs from the same donors including cell viability and properties such as cell attachment, spreading and tight junction formation, extending our previous work. At the transcriptional level, upregulation of BMI-1 in HBECs affected global gene expression, equivalent to $1.5 \%$ of transcripts tested and provide a useful dataset prior to utilising these cells for specific research questions. Our evaluation of the B-HBEC platform specifically for asthma showed that $97.6 \%$ of genes implicated in asthma were not affected by BMI-1 overexpression, supporting the use of the platform in asthma research. Overall, these data provide the first in-depth characterisation of this research platform, supporting its utility in asthma research where the airway epithelium has been implicated in disease mechanisms and where obtaining cell samples from control subjects and asthma patients comes with associated risk.

There have been a number of attempts to enhance the replicative lifespan of HBECs predominantly through viral oncoproteins such as SV40 large T-antigen transformation $[18,19]$, or HPV-16 [20]. Alternatively, overexpression of hTERT alone has been shown to successfully delay cell senescence [21]. Whilst several of these cell lines retain key epithelial cell properties, they do have limitations (e.g. hTERT cells lose the ability to form a resistive barrier and have chromosomal abnormalities) $[10,21]$.

The transcriptional repressor, BMI-1, is part of the polycomb repressive complex-1 and one of the target genes of this complex is thought to be CDKN2A ( $16^{\mathrm{INK} 4 \mathrm{~A}}$ ) [22]. We have confirmed downregulation of p16 protein previously [10] and now show that B-HBECs progress through the G1/S checkpoint more readily compared to N-HBECs. Downregulation of CDKN2A was observed in our differential gene expression analysis (mean FPKM 32, (range 21-46) in N-HBEC versus mean FPKM 20, (range 9-26) in $\mathrm{B}-\mathrm{HBEC}$ ); however, this did not reach statistical significance.

BMI-1 is implicated in a number of different cancers largely due to its role in stem-cell renewal but also its regulation of proliferation [23-25]. Collectively, when we compared the cell viability of N-HBECs and B-HBECs, no statistically significant difference was observed, although individually $5 / 6$ B-HBEC donors showed an increase. We also observed no difference in cell viability between N-HBECs and B-HBECs, although cell viability was high $(>85 \%)$ in each cell line.

ECIS has previously been shown to be a sensitive and noninvasive technique for measuring multiple in vitro properties of bronchial epithelial cells [26]. We showed that N-HBECs and B-HBECs attach and spread in a highly similar manner. Previous studies have found that upregulation of BMI-1 results in the downregulation of E-cadherin and the upregulation of EMT markers [27-29]. One limitation of ECIS is that cells are cultured in an undifferentiated state and therefore not representative of in vivo conditions. However, we have previously shown that differentiated B-HBECs develop a resistive barrier comparable to N-HBECs when cultured at ALI [10], indicating that a robust epithelial barrier model can be achieved. This is particularly of relevance to asthma, as bronchial epithelial cells isolated from asthma patients have defective barrier properties [3] and the BMI-1 platform may be useful to extend the lifespan of patient cells for mechanistic studies.

Previous studies that have manipulated BMI-1 expression have shown widespread changes to gene expression as a consequence [30]. BMI-1 $1^{-1-}$ in neural progenitor cells resulted in over 500 genes being upregulated alone [31]. We identified 293 genes to be differentially expressed due to BMI-1 upregulation (5\% FDR). Although a significant number, a comparison of gene expression between in vivo airway epithelia and differentiated airway epithelial cells cultured at the ALI, found 453 genes that were differentially expressed with a more stringent $<1 \%$ FDR [32]. Whilst BMI-1 expression did not result in widespread changes in expression to key epithelial cell markers, significantly, BMI-1 downregulated transcripts that are associated with epithelial differentiation pathways (KRT4, KRT13, SPINK5, CEACAM5/ 6, SPRR1A and SPRR1B). KRT4 and KRT13 are markers of a newly identified transitional cell identified in the mouse airway [33], a finding which requires further investigation.

We observed many pathways $(>150)$ that were significantly altered by BMI-1. The most upregulated pathway in B-HBECs consisted of genes involved in EGFR signalling, a pathway that has previously been 
associated with chronic airway diseases such as asthma $[34,35]$. The regulatory role that BMI-1 has in the cell cycle has been well characterised and it was therefore anticipated that two of the most enriched pathways in B-HBECs related to cell cycle genes [36, 37]. BMI-1 expression also increased expression of genes associated with cell proliferation and EMT; these findings are supported by the literature linking BMI-1 to multiple different cancers $[24,25,38,39]$. This characterisation provides information for researchers using this platform.

The use of BMI-1 to expand the replicative lifespan of HBECs will potentially allow us to capture the phenotype of HBECs derived from bronchial brushes for a range of respiratory disease patients including asthma. Our data from these analyses indicate that the upregulation of BMI-1 does not reduce inter-donor variability. Furthermore, BMI-1 has previously been utilised to delay cell senescence in cystic fibrosis cells, whilst retaining their disease phenotype [13]. The extended lifespan of these cells gives researchers more scope for experiments such as CRISPR/cas9 gene editing and single cell cloning prior to ALI expansion. This is challenging in primary airway cells as ALI is required to be performed on early passage cells, limiting the capacity of the cells to undergo manipulation prior to ALI.

GWAS and RNA-sequencing studies of asthma have identified multiple candidate genes linked to disease and we found that the majority of these (97.6\%) were not influenced by BMI-1 overexpression, supporting the use of this platform. Seven of these genes were significantly differentially regulated by BMI- 1 over expression (downregulated: SERPINB7, CYP2J2, UPK1B, SNTB1 and CEACAM5; upregulated: ITGB6 and EFNB2).

To avoid the effects of prolonged BMI-1 expression we also produced an inducible BMI-1 HBEC platform, regulated by a cumate switch system. We observed significant upregulation of BMI-1 in IB-HBECs cultured in cumate-supplemented medium, although more heterogeneous than the constitutive system. When cumate was withdrawn from the culture medium, BMI-1 expression did not return to basal levels observed in N-HBECs, although some donors did show a reduction. This finding, further supported by the limited concordance in B-HBEC and IB-HBEC transcriptomes, suggests that the inducible platform has limited utility.

The major limitation of this study is that we did not overexpress BMI-1 in cells isolated from asthma patients to compare to healthy controls, however this was beyond the primary aim of the present study. We have previously shown that BMI-1 HBECs maintain the ability to differentiate and form an epithelial barrier at ALI; however, it is as yet unclear as to whether BMI-1-overexpressing cells derived from asthma patients would exhibit an altered phenotype at the ALI [10]. It is also important to note that several changes observed in the B-HBECs are relevant to asthma phenotypes including changes to matrix proteins, mucociliary differentiation, EGFR signalling and EMT $[35,40]$. Therefore, additional caution and characterisation is required prior to B-HBECs being confirmed as a suitable cell model for asthma mechanisms.

In conclusion, this study highlights the utility of the constitutive BMI-1 platform for capturing and studying the phenotype of airway epithelial cells, whilst retaining interdonor variability. This provides a platform to investigate disease mechanisms over prolonged periods and reducing reliance on bronchial brushes and biopsies, procedures that are invasive and present a risk for patients with airway disease. Finally, the extended lifespan of cells will provide a platform for more challenging mechanistic studies that may require frequent cell passaging (e.g. CRISPR/Cas9 gene and variant editing).

Author Contributions: I. Sayers conceived and designed the study. T.V. Sharp contributed to the design of the study. J. O'Loughlin and R. Hall designed and completed experiments, and analysed data. S. Bhaker, M.A. Portelli and A. Henry performed experiments and analysed data. V. Pang and D.O. Bates facilitated the design of ECIS experiments. I. Sayers, J. O'Loughlin and R. Hall wrote the paper with contributions from all authors.

Conflict of interest: J. O'Loughlin has nothing to disclose. R. Hall has nothing to disclose. S. Bhaker has nothing to disclose. M.A. Portelli has nothing to disclose. A. Henry has nothing to disclose. V. Pang has nothing to disclose. D.O. Bates has nothing to disclose. T.V. Sharp has nothing to disclose. I. Sayers reports grants from Asthma UK, the Hermes Fellowship and the British Lung Foundation during the conduct of the study and grants from GSK and Boehringer Ingelheim outside the submitted work.

Support statement: This study was supported by Asthma UK (grants 10/006 and 11/031), a Hermes Fellowship (University of Nottingham, 2016-2017), the British Lung Foundation (grant PPRG15-5) and the British Heart Foundation (grant PG/13/85/30536). Funding information for this article has been deposited with the Crossref Funder Registry.

\section{References}

1 Nicodemus-Johnson J, Myers RA, Sakabe NJ, et al. DNA methylation in lung cells is associated with asthma endotypes and genetic risk. JCI Insight 2016; 1: e90151-e. 
2 Parker J, Sarlang S, Thavagnanam S, et al. A 3-D well-differentiated model of pediatric bronchial epithelium demonstrates unstimulated morphological differences between asthmatic and nonasthmatic cells. Pediatr Res 2010; 67: $17-22$.

3 Xiao C, Puddicombe SM, Field S, et al. Defective epithelial barrier function in asthma. J Allergy Clin Immunol 2011; 128: 549-556.

4 Shrine N, Portelli MA, John C, et al. Moderate-to-severe asthma in individuals of European ancestry: a genome-wide association study. Lancet Respir Med 2019; 7: 20-34.

5 Pividori M, Schoettler N, Nicolae DL, et al. Shared and distinct genetic risk factors for childhood-onset and adult-onset asthma: genome-wide and transcriptome-wide studies. Lancet Respir Med 2019; 7: 509-522.

6 Loffredo LF, Abdala-Valencia H, Anekalla KR, et al. Beyond epithelial-to-mesenchymal transition: Common suppression of differentiation programs underlies epithelial barrier dysfunction in mild, moderate, and severe asthma. Allergy 2017; 72: 1988-2004.

7 Stewart CE, Torr EE, Mohd Jamili NH, et al. Evaluation of differentiated human bronchial epithelial cell culture systems for asthma research. J Allergy (Cairo) 2012; 2012: 943982.

8 Liu X, Ory V, Chapman S, et al. ROCK inhibitor and feeder cells induce the conditional reprogramming of epithelial cells. Am J Pathol 2012; 180: 599-607.

9 García-Escudero V, García-Gómez A, Gargini R, et al. Prevention of senescence progression in reversibly immortalized human ensheathing glia permits their survival after deimmortalization. Mol Ther 2010; 18: 394-403.

10 Torr E, Heath M, Mee M, et al. Expression of polycomb protein BMI-1 maintains the plasticity of basal bronchial epithelial cells. Physiol Rep 2016; 4: e12847.

11 Itahana $\mathrm{K}$, Zou Y, Itahana $\mathrm{Y}$, et al. Control of the replicative life span of human fibroblasts by p16 and the polycomb protein Bmi-1. Mol Cell Biol 2003; 23: 389-401.

12 Jacobs JJL, Kieboom K, Marino S, et al. The oncogene and polycomb-group gene bmi-1 regulates cell proliferation and senescence through the ink4a locus. Nature 1999; 397: 164-168.

13 Munye MM, Shoemark A, Hirst RA, et al. BMI-1 extends proliferative potential of human bronchial epithelial cells while retaining their mucociliary differentiation capacity. Am J Physiol Lung Cell Mol Physiol 2017; 312 L258-L267.

14 Wegener J, Keese CR, Giaever I. Electric cell-substrate impedance sensing (ECIS) as a noninvasive means to monitor the kinetics of cell spreading to artificial surfaces. Exp Cell Res 2000; 259: 158-166.

15 Watson JV, Chambers SH, Smith PJ. A pragmatic approach to the analysis of DNA histograms with a definable G1 peak. Cytometry 1987; 8: 1-8.

16 Trapnell C, Roberts A, Goff L, et al. Differential gene and transcript expression analysis of RNA-seq experiments with TopHat and Cufflinks. Nat Protoc 2012; 7: 562-578.

17 Kheirallah AK, de Moor CH, Faiz A, et al. Lung function associated gene integrator complex subunit 12 regulates protein synthesis pathways. BMC Genomics 2017; 18: 248.

18 Zeitlin PL, Lu L, Rhim J, et al. A cystic fibrosis bronchial epithelial cell line: immortalization by adeno-12-SV40 infection. Am J Respir Cell Mol Biol 1991; 4: 313-319.

19 Cozens AL, Yezzi MJ, Kunzelmann K, et al. CFTR expression and chloride secretion in polarized immortal human bronchial epithelial cells. Am J Respir Cell Mol Biol 1994; 10: 38-47.

20 Willey JC, Broussoud A, Sleemi A, et al. Immortalization of normal human bronchial epithelial cells by human papillomaviruses 16 or 18. Cancer Res 1991; 51: 5370-5377.

21 Piao CQ, Liu L, Zhao YL, et al. Immortalization of human small airway epithelial cells by ectopic expression of telomerase. Carcinogenesis 2005; 26: 725-731.

22 Rayess H, Wang MB, Srivatsan ES. Cellular senescence and tumor suppressor gene p16. Int J Cancer 2012; 130: 1715-1725.

23 Srinivasan M, Bharali DJ, Sudha T, et al. Downregulation of Bmil in breast cancer stem cells suppresses tumor growth and proliferation. Oncotarget 2017; 8: 38731-38742.

$24 \mathrm{Wu} \mathrm{C}$, Zheng $\mathrm{X}, \mathrm{Li} \mathrm{X}$, et al. Reduction of gastric cancer proliferation and invasion by miR-15a mediated suppression of Bmi-1 translation. Oncotarget 2016; 7: 14522-14536.

25 Zhu S, Zhao D, Yan L, et al. BMI1 regulates androgen receptor in prostate cancer independently of the polycomb repressive complex 1. Nat Commun 2018; 9: 500.

26 Heijink IH, Brandenburg SM, Noordhoek JA, et al. Characterisation of cell adhesion in airway epithelial cell types using electric cell-substrate impedance sensing. Eur Respir J 2010; 35: 894-903.

27 Song LB, Li J, Liao WT, et al. The polycomb group protein Bmi-1 represses the tumor suppressor PTEN and induces epithelial-mesenchymal transition in human nasopharyngeal epithelial cells. J Clin Invest 2009; 119: 3626-3636.

28 Paranjape AN, Balaji SA, Mandal T, et al. Bmil regulates self-renewal and epithelial to mesenchymal transition in breast cancer cells through Nanog. BMC Cancer 2014; 14: 785.

29 Dong P, Kaneuchi M, Watari H, et al. MicroRNA-194 inhibits epithelial to mesenchymal transition of endometrial cancer cells by targeting oncogene BMI-1. Mol Cancer 2011; 10: 99.

30 Ganapathi M, Boles NC, Charniga C, et al. Effect of Bmil over-expression on gene expression in adult and embryonic murine neural stem cells. Sci Rep 2018; 8: 7464.

31 Gargiulo G, Cesaroni M, Serresi M, et al. In vivo RNAi screen for BMI1 targets identifies TGF-beta/BMP-ER stress pathways as key regulators of neural- and malignant glioma-stem cell homeostasis. Cancer Cell 2013; 23 660-676.

32 Pezzulo AA, Starner TD, Scheetz TE, et al. The air-liquid interface and use of primary cell cultures are important to recapitulate the transcriptional profile of in vivo airway epithelia. Am J Physiol Lung Cell Mol Physiol 2011; 300: L25-L31.

33 Montoro DT, Haber AL, Biton M, et al. A revised airway epithelial hierarchy includes CFTR-expressing ionocytes. Nature 2018; 560: 319-324.

34 Puddicombe SM, Polosa R, Richter A, et al. Involvement of the epidermal growth factor receptor in epithelial repair in asthma. FASEB J 2000; 14: 1362-1374

35 Heijink $\mathrm{IH}$, van Oosterhout A, Kapus A. Epidermal growth factor receptor signalling contributes to house dust mite-induced epithelial barrier dysfunction. Eur Respir J 2010; 36: 1016-1026. 
Lee K, Adhikary G, Balasubramanian S, et al. Expression of Bmi-1 in epidermis enhances cell survival by altering cell cycle regulatory protein expression and inhibiting apoptosis. J Invest Dermatol 2008; 128: 9-17.

37 Park IK, Qian D, Kiel M, et al. Bmi-1 is required for maintenance of adult self-renewing haematopoietic stem cells. Nature 2003; 423: 302-305.

38 Yuan W, Yuan Y, Zhang T, et al. Role of Bmi-1 in regulation of ionizing irradiation-induced epithelial-mesenchymal transition and migration of breast cancer cells. PLoS One 2015; 10: e0118799.

39 Yong KJ, Basseres DS, Welner RS, et al. Targeted BMI1 inhibition impairs tumor growth in lung adenocarcinomas with low CEBPalpha expression. Sci Transl Med 2016; 8: 350ra104.

40 Bosco A, Ehteshami S, Stern DA, et al. Decreased activation of inflammatory networks during acute asthma exacerbations is associated with chronic airflow obstruction. Mucosal Immunol 2010; 3: 399-409. 